\title{
Absence of Interaction as a Consequence of Good Ultraviolet Behavior in the Case of a Local Fermi Field
}

\author{
Robert T. Powers* \\ Palmer Physical Laboratory, Princeton University, Princeton, New Jersey
}

Received June 1, 1966

\begin{abstract}
The ICAR theorem asserts that a local relativistic Fermi field is necessarily a free field, if it satisfies the canonical anticommutation relations, irreducibility of the fields at a fixed time, and certain regularity conditions. The regularity conditions are slightly stronger than the requirement that the mass renormalization be finite. It follows that an interacting Fermi field must violate one or more assumptions of the ICAR theorem.
\end{abstract}

\section{The ICAR theorem}

We prove a theorem which demonstrates the intrinsic difficulties associated with the construction of a relativistic quantum field theory satisfying an irreducible representation of the canonical anticommutation relations. The theorem will be called the ICAR theorem. The ICAR theorem asserts that a local relativistic Fermi field is necessarily a free field, if it satisfies the canonical anticommutation relations, irreducibility of the fields at a fixed time, and certain regularity conditions. The regularity conditions are slightly stronger than the requirement that the mass renormalization be finite. It follows that an interacting Fermi field must violate one or more assumptions of the ICAR theorem.

A Fermi field will violate the assumptions of the ICAR theorem if either the mass renormalization or field strength renormalization is infinite. It is well known that the perturbation expansion for interacting Fermi fields implies that the mass and field strength renormalizations are infinite. The ICAR theorem suggests that the divergence of the renormalization constants is intrinsic to interacting Fermi fields and is not particular to the perturbation theory approach. This contention is similar to but weaker than KäLLÉN's contention [1], that one of the renormalization constants in quantum electrodynamics is infinite.

Even if the renormalization constants are finite, a Fermi field may fail to satisfy the assumptions of the ICAR theorem because the fields at a fixed time are reducible. This means there are non-trivial operators which commute with the fields at a fixed time. In this sense the ICAR

* Work supported in part by the U.S. Air Force Office of Research, Air Research and Development Command under contract number AF 49(638)-1545.

11 Commun. math. Phys., Vol. 4 
theorem supports KLAUDER's contention [2] that one should use reducible representations of the canonical variables for the construction of interacting fields. It may be that the difficulties encountered in perturbation theory are a result of trying to solve the field equations in the context of an irreducible representation of the canonical variables, when a reducible representation would be more appropriate.

The ICAR theorem follows from assumptions I-IV, as stated in the next section.

\section{Assumptions}

(I) $\psi$ is a local relativistic Fermi field in the sense of the Wightman framework [3]. Briefly, this assumption means the following: $\psi$ is an operator valued distribution in a separable Hilbert space, $\mathfrak{H}$. The smeared fields transform according to a unitary representation of the inhomogeneous Lorentz group. There is a unique vacuum state, $\Omega_{0} \in \mathfrak{F}$, invariant under inhomogeneous Lorentz transformations and cyclic in $\mathfrak{G}$ with respect to the smeared fields. The spectrum of the translation operator is assumed to lie in the forward light cone. The field is local, i.e.,

$$
\begin{aligned}
\left\{\psi_{r}(x), \psi_{s}(y)\right\}_{+} & =0 \\
\left\{\psi_{r}(x)^{*}, \psi_{s}(y)\right\}_{+} & =0
\end{aligned} \quad \text { if } \quad(x-y)^{2}<0
$$

where $\{A, B\}_{+}=A B+B A$ and $(x-y)^{2}=\left(y_{0}-y_{0}\right)^{2}-(\mathbf{x}-\mathbf{y})^{2} \cdot \psi$ is an $m$-component Fermi field. The components label the various types of particles described by the field, and their spin components. We assume the number of space-time dimensions, $n+1$, is not less than three (i.e., $n \geqq 2$ ).

(II) $\psi$ satisfies the canonical anticommutation relations (CAR). If this assumption is to have meaning, one must assume that the fields make sense when smeared with test functions at a fixed time. We denote by $\psi(f, t)$ and $\psi(f, t)^{*}$ the smeared field and its hermitian adjoint at a fixed time, $t$. The fields, $\psi(f, t)$ and $\psi(f, t)^{*}$, are, respectively, linear and antilinear operator functions of the test function, $f$. The CAR expressed in terms of the smeared fields are

$$
\begin{gathered}
\{\psi(f, t), \psi(g, t)\}_{+}=0, \\
\left\{\psi(f, t)^{*}, \psi(g, t)\right\}_{+}=(f, g)=\int \sum_{r=1}^{m} \overline{f_{r}(\mathbf{x})} g_{r}(\mathbf{x}) d^{n} \mathbf{x} .
\end{gathered}
$$

Because of the CAR, the smeared field operators at a fixed time are bounded by the $L^{2}$-norm of the test function [4]. The norm of the smeared field operators is given by the expression,

$$
\|\psi(f, t)\|=\|\psi(f, t) *\|=\sqrt{(f, f)}=\|f\| .
$$


Therefore, we can assume, without loss of generality, that the smeared fields, $\psi(f, t)$ and $\psi(f, t)^{*}$, are defined for all square integrable $m$-component test functions, $f \in \mathfrak{G}_{T}$. The test function space, $\mathfrak{G}_{T}$, is a Hilbert space of test functions.

(III) The smeared fields at a fixed time act irreducibly on $\mathfrak{G}$. This assumption implies that every bounded operator, $A$, on $\mathfrak{S}$ which commutes with the field operators, $\psi(f, t)$ and $\psi(f, t)^{*}$, at time, $t$, for all $f \in \mathfrak{S}_{T}$, is a multiple of the identity. Since the fields at time, $t$, are related to the fields at time, $t=0$, by a unitary transformation, $(\psi(f, t)$ $\left.=U(t) \psi(f, 0) U(t)^{-1}\right)$, irreducibility of the fields at time, $t=0$, implies irreducibility of the fields at all times.

(IV) The time derivatives of the fields can be applied to the vacuum and to single particle states. Explicitly we assume that the expressions,

$$
\begin{gathered}
\lim _{t \rightarrow 0}\left(\frac{\psi(f, t)^{\#}-\psi(f, 0) \#}{t}\right) \Omega_{0}=\partial_{t} \psi(f, 0)^{\#} \Omega_{0}, \\
\lim _{t \rightarrow 0}\left(\frac{\psi(f, t)^{\#}-\psi(f, 0)^{\#}}{t}\right) \psi(g, 0)^{\#} \Omega_{0}=\left(\partial_{t} \psi(f, 0)^{\#}\right) \psi(g, 0)^{\#} \Omega_{0},
\end{gathered}
$$

converge strongly to a limit as $t \rightarrow 0$, provided the test functions, $f$ and $g$, are in Schwartz's space, $\mathfrak{T}$, of infinitely differentiable functions of fast decrease. The notation, $\psi(f, t)^{\#}$, stands for either $\psi(f, t)$ or $\psi(f, t)^{*}$.

The question of whether the limit (4) exists can be expressed in terms of the two-point vacuum expectation function. We consider, for an example, a Fermi-Dirac field which is invariant under charge conjugation. The two-point function can be written, using the Lehmann spectral representation, in the form [5],

$$
\begin{aligned}
& \left(\Omega_{0}, \psi(x) \psi(y)^{+} \Omega_{0}\right)= \\
& \int_{0}^{\infty} d m^{2}\left\{\varrho_{1}\left(m^{2}\right)\left(-i S^{(+)}(x-y ; m)\right)+\varrho_{2}\left(m^{2}\right) i \Delta^{(+)}\left(x-y ; m^{2}\right)\right\}
\end{aligned}
$$

with $2 m \varrho_{1}\left(m^{2}\right) \geqq \varrho_{2}\left(m^{2}\right) \geqq 0$ and $\psi^{+}=\psi^{*} \gamma^{0}$.

The expressions, $\varrho_{1}\left(m^{2}\right) d m^{2}$ and $\varrho_{2}\left(m^{2}\right) d m^{2}$, are positive measures. Since the field satisfies the CAR, the spectral measure, $\varrho_{1}$, satisfies the condition,

$$
\int_{0}^{\infty} \varrho_{1}\left(m^{2}\right) d m^{2}=1
$$

A straightforward computation shows that limit (4) exists if and only if,

$$
\int_{0}^{\infty} m^{2} \varrho_{1}\left(m^{2}\right) d m^{2}<\infty
$$

This condition is stronger than the requirement that the mass renormalization be finite. The mass renormalization is finite if the first moment of $m$ is finite, with respect to $\varrho_{1}\left(m^{2}\right) d m^{2}$. 
The question of the existence of limit (5) can be determined from the properties of the four-point functions.

\section{Proof of the ICAR theorem}

Theorem. If $\psi$ is a Fermi field satisfying assumptions $I-I V$, then $\psi$ is a free field in the sense that $\psi$ satisfies the linear differential equation,

$$
\partial_{t} \psi(f, t)=\psi\left(T_{1} f, t\right)+\psi\left(T_{2} f, t\right)^{*}, \quad f \in \mathfrak{T},
$$

where $T_{1}$ and $T_{2}$ are, respectively, linear and antilinear operators defined on $\mathfrak{T}$. The time derivative of the field exists in the sense of strong convergence, i.e., the limit,

$$
\lim _{h \rightarrow 0}\left(\frac{\psi(f, t+h)-\psi(f, t)}{h}\right) \Omega=\partial_{t} \psi(f, t) \Omega, \quad f \in \mathfrak{T},
$$

exists for all $\Omega \in \mathfrak{S}$.

The theorem will be proven in steps by the following lemmas.

It is clear that equation (6) implies the field is non-interacting. It follows from this equation, that the fields at time, $t$, are related to the fields at time, $t=0$, by a Bogoliubov transformation, i.e.,

$$
\psi(f, t)=\psi\left(S_{1}(t) f, 0\right)+\psi\left(S_{2}(t) f, 0\right) *,
$$

where $S_{1}(t)$ and $S_{2}(t)$ are, respectively, bounded linear and antilinear operators defined on the test function space, $\mathfrak{H}_{T}$. The Bogoliubov transformation defined in equation (7) propagates the fields in accordance with the field equation (6). These Bogoliubov transformations are the oneparameter group of transformations generated by the infinitesimal operators $T_{1}$ and $T_{2}$, i.e.,

$$
\begin{aligned}
& T_{1}=\lim _{t \rightarrow 0} \frac{S_{1}(t)-1}{t}, \\
& T_{2}=\lim _{t \rightarrow 0} \frac{S_{2}(t)}{t} .
\end{aligned}
$$

We do not take on the problem of characterizing the form of the operators, $T_{1}$ and $T_{2}$. Clearly, this problem is equivalent to the problem of characterizing all possible relativistic free field equations which are consistent with the CAR. We remark that for a gauge invariant theory of a Fermi-Dirac field, equation (6) is simply the statement of the Dirac equation, i.e.,

$$
T_{\mathbf{1}}=-i(\boldsymbol{\alpha} \cdot \mathbf{p}+\beta m), \quad T_{\mathbf{2}}=0 .
$$

We now proceed with the proof of the ICAR theorem. The heart of the proof is contained in lemma 1. For the proof of this lemma we only assume that the field is local and that the field satisfies the CAR.

Lemma 1. Suppose $f, g, h \in \mathfrak{H}_{T}$ are square integrable m-component functions of $\mathbf{x}=\left(x_{1}, \ldots, x_{n}\right)$. And furthermore, suppose the functions $f$ and 
$g$ are bounded functions of $\mathbf{x}$, so that $\left|f_{r}(\mathbf{x})\right| \leqq C_{1}$ and $\left|g_{r}(\mathbf{x})\right| \leqq C_{2}$ for all $\mathbf{x}$ and $r=1, \ldots, m$. Then, if $\psi$ is a local relativistic field satisfying the $C A R$, the following inequality holds:

$$
\left\|\left[\psi(h, 0)^{\#,}\left\{\psi(g, 0)^{\#}, \psi(f, t)^{\#}\right\}_{+}\right]_{-}\right\| \leqq K C_{1} C_{2}|t|^{n}\|h\|,
$$

where $K$ is a constant depending only on the integers, $n$ and $m$.

Proof. We define $Z(h, g, f ; t)$ by the relation,

$$
Z(h, g, f ; t)=\left[\psi(h, 0)^{\#},\left\{\psi(g, 0)^{\#}, \psi(f, t)^{\#}\right\}_{+}\right]_{-} .
$$

Since the anticommutator of $\psi(h, 0)^{\#}$ and $\psi(g, 0)^{\#}$ is a c-number (a multiple of the identity) the expression for $Z$ is antisymmetric under the interchange of $\psi(h, 0)^{\#}$ and $\psi(g, 0)^{\#}$, i.e.,

$$
Z(h, g, f ; t)=-\left[\psi(g, 0)^{\#},\left\{\psi(h, 0)^{\#}, \psi(f, t)^{\#}\right\}_{+}\right]_{-} .
$$

We denote by $\operatorname{supp}\left(f ; t_{0}\right)$ (the support of $f$ on the time, $t=t_{0}$, plane) the set of space-time points, $(\mathbf{x}, t)$, such that $t=t_{0}$ and $f_{r}(\mathbf{x}) \neq 0$ for some $r=1, \ldots, m$. Since $\psi$ is assumed to be a local field, the anticommutator, $\left\{\psi(g, 0)^{\#}, \psi(f, t)^{\#}\right\}_{+}$, vanishes if the sets, $\operatorname{supp}(f ; t)$ and $\operatorname{supp}(g ; 0)$, are space-like separated. Therefore, $Z(h, g, f ; t)$ vanishes if the sets, $\operatorname{supp}(f ; t)$ and $\operatorname{supp}(g ; 0)$, are space-like separated. And from equation (1a) it follows that $Z(h, g, f ; t)$ vanishes if $\operatorname{supp}(f ; t)$ and $\operatorname{supp}(h ; 0)$ are spacelike separated. We will use this result to estimate the norm of $Z$.

Consider the time, $t$, occurring in the definition of $Z$ to have a fixed non-zero value. We consider subdividing the $t=0$ plane into cells of edge length, $t$. The cells are labelled by lattice vectors, $\mathrm{k}$, with integer components,

$$
S_{\mathbf{k}}=\left\{\mathbf{x} \mid t k_{i} \leqq x_{i}<t\left(k_{i}+1\right) ; i=1, \ldots, n\right\} .
$$

The points, $t \mathbf{k}=\left(t k_{1}, \ldots, t k_{n}\right)$, lie on the corners of the cells, $S_{\mathbf{k}}$. Let $E_{\mathbf{k}}$ be the hermitian projection onto the space of functions with support in $S_{\mathbf{k}}$, i.e.,

$$
\begin{aligned}
\left(E_{\mathbf{k}} f\right)_{r}(\mathbf{x}) & =f_{r}(\mathbf{x}) & & \mathbf{x} \in S_{\mathbf{k}}, \\
& =0 & & \mathbf{x} \notin S_{\mathbf{k}} .
\end{aligned}
$$

Since $Z(h, g, f ; t)$ is linear or antilinear in each of its arguments, $h, g$, and $f$, it follows that

$$
\begin{aligned}
Z(h, g, f ; t) & =\sum_{\mathbf{k} \mathbf{l} \mathbf{r}} Z\left(E_{\mathbf{k}} h, E_{\mathbf{l}} g, E_{\mathbf{r}} f ; t\right) \\
& =\sum_{\mathbf{k} \mathbf{l}} Z(\mathbf{k}, \mathbf{l}, \mathbf{r}) .
\end{aligned}
$$

The sum converges in norm because the field operators are bounded by the $L^{2}$-norm of the test function. We define the hermitian operator, $Q(h, g, f ; t)$, as follows:

$$
Q(h, g, f ; t)=\frac{\alpha Z(h, g, f ; t)+\bar{\alpha} Z(h, g, f ; t)^{*}}{2} \text { where }|\alpha|=1
$$


and

$$
Q(\mathbf{k}, \mathbf{l}, \mathbf{r})=\frac{1}{2}\left(\alpha Z(\mathbf{k}, \mathbf{l}, \mathbf{r})+\bar{\alpha} Z(\mathbf{k}, \mathbf{l}, \mathbf{r})^{*}\right) .
$$

We will estimate the norm of $Q^{2}$, using the equation,

$$
Q^{2}=\sum_{\mathbf{k}^{\prime} s \mathbf{1}^{\prime} s} Q\left(\mathbf{k}_{1}, \mathbf{k}_{2}, \mathbf{k}_{3}\right) Q\left(\mathbf{l}_{1}, \mathbf{l}_{2}, \mathbf{l}_{3}\right) .
$$

Two lattice vectors, $\mathbf{k}$ and $\mathbf{l}$ are said to be adjacent if $\left|k_{i}-l_{i}\right| \leqq 1$ for each $i=1, \ldots, n$. Every lattice vector has $3^{n}$ adjacent lattice vectors, including itself. Due to the way the cells, $S_{\mathbf{k}}$, were defined, the sets, $\operatorname{supp}\left(E_{\mathbf{k}} f ; t\right)$ and $\operatorname{supp}\left(E_{1} g ; 0\right)$, are space-like separated if $\mathbf{k}$ and $\mathbf{l}$ are not adjacent. Hence, we have

$$
\left\{\psi\left(E_{\mathbf{k}} f, t\right)^{\#,} \psi\left(E_{1} g, 0\right)^{\#}\right\}_{+}=0,
$$

if $\mathbf{k}$ and $\mathbf{l}$ are not adjacent.

Therefore, $Q\left(\mathbf{k}_{1}, \mathbf{k}_{2}, \mathbf{k}_{3}\right) \neq 0$ only if $\mathbf{k}_{1}$ and $\mathbf{k}_{3}$ are adjacent and $\mathbf{k}_{2}$ and $\mathbf{k}_{3}$ are adjacent. Then, the sum for $Q^{2}$ can be written,

$$
Q(h, g, f ; t)^{2}=\sum_{\mathbf{r s}} \sum_{\mathbf{k}_{1} \mathbf{k}_{2}}^{(r)} \sum_{\mathbf{l}_{1} \mathbf{l}_{2}}^{(s)} Q\left(\mathbf{k}_{1}, \mathbf{k}_{2}, \mathbf{r}\right) Q\left(\mathbf{l}_{1}, \mathbf{l}_{2}, \mathbf{s}\right),
$$

where $\sum_{\mathbf{k}_{1} \mathbf{k}_{2}}^{(r)}$ is the sum over all $\mathbf{k}_{1}$ and $\mathbf{k}_{2}$ adjacent to $\mathbf{r}$.

It follows from relation (lc) that $Q\left(\mathbf{k}_{1}, \mathbf{k}_{2}, \mathbf{k}_{3}\right)$ anticommutes with $Q\left(\mathbf{l}_{1}, \mathbf{l}_{2}, \mathbf{l}_{3}\right)$, if none of the k's are adjacent to any of the $\mathbf{l}^{\prime}$ s, i.e.,

$$
\left\{Q\left(\mathbf{k}_{1}, \mathbf{k}_{2}, \mathbf{k}_{3}\right), Q\left(\mathbf{l}_{1}, \mathbf{l}_{2}, \mathbf{l}_{3}\right)\right\}_{+}=0,
$$

if $\mathbf{k}_{i}$ is not adjacent to $\mathbf{l}_{j} ; i, j=1,2,3$.

Therefore, many of the "off diagonal" terms in the sum for $Q^{2}$ cancel. The terms which survive are of the form,

$$
Q(h, g, f ; t)^{2}=\Sigma_{\mathbf{r s}}^{\prime} \sum_{\mathbf{k}_{1} \mathbf{k}_{2}}^{(r)} \sum_{\mathbf{l}_{1} \mathbf{l}_{2}}^{(s)} Q\left(\mathbf{k}_{1}, \mathbf{k}_{2}, \mathbf{r}\right) Q\left(\mathbf{l}_{1}, \mathbf{l}_{2}, \mathbf{s}\right),
$$

where $\sum_{\mathrm{r} s}^{\prime}$ is the sum over all $\mathrm{r}$ and $\mathrm{s}$ such that $\left|r_{i}-s_{i}\right| \leqq 2$, for $i=1, \ldots, n$. If $\left|r_{i}-s_{i}\right|>2$ for some $i$, then

$\sum_{\mathbf{k}_{1} \mathbf{k}_{2}}^{(r)} \sum_{\mathbf{l}_{1} \mathbf{l}_{2}}^{(s)} Q\left(\mathbf{k}_{1}, \mathbf{k}_{2}, \mathbf{r}\right) Q\left(\mathbf{l}_{1}, \mathbf{l}_{2}, \mathrm{~s}\right)+\sum_{\mathbf{k}_{1} \mathbf{k}_{2}}^{(r)} \sum_{\mathbf{l}_{1} \mathbf{l}_{2}}^{(s)} Q\left(\mathbf{k}_{1}, \mathbf{k}_{2}, \mathbf{s}\right) Q\left(\mathbf{l}_{1}, \mathbf{l}_{2}, \mathbf{r}\right)=0$

because of equation (ld).

It follows from the fact that the field operators are bounded by the $L^{2}$-norm of the test functions (equation (3)) that

$$
\|Q(h, g, f ; t)\| \leqq 4\|h\|\|g\|\|f\| .
$$

Since $f$ and $g$ are bounded functions of $\mathbf{x}$, so that $\left|f_{r}(\mathbf{x})\right| \leqq C_{1}$ and $\left|g_{r}(\mathbf{x})\right| \leqq C_{2}$, the $L^{2}$-norms of $E_{\mathbf{k}} f$ and $E_{1} g$ are bounded by the expressions,

$$
\left\|E_{\mathbf{k}} f\right\| \leqq C_{1} \sqrt{m|t|^{n}},\left\|E_{1} g\right\| \leqq C_{2} \sqrt{m|t|^{n}} .
$$

Therefore, it follows that

$$
\left\|Q\left(\mathbf{k}_{1}, \mathbf{k}_{2}, \mathbf{k}_{3}\right)\right\| \leqq 4 C_{1} C_{2} m|t|^{n}\left\|E_{\mathbf{k}_{1}} h\right\| .
$$


Using relations (le) and (lf) we estimate the norm of $Q^{2}$ :

$$
\begin{aligned}
&\left\|Q^{2}\right\| \leqq \sum_{\mathrm{rs}}^{\prime} \sum_{\mathbf{k}_{1} \mathbf{k}_{2}}^{(r)} \sum_{\mathbf{l}_{1} \mathbf{1}_{2}}^{(s)} 16 C_{1}^{2} C_{2}^{2} m^{2}|t|^{2 n}\left\|E_{\mathrm{r}} h\right\|\left\|E_{\mathrm{s}} h\right\| \\
& \leqq \sum_{\mathrm{r} \mathbf{s}}^{\prime} 16 \cdot 3^{4 n} C_{1}^{2} C_{2}^{2} m^{2}|t|^{2 n}\left\|E_{\mathrm{r}} h\right\|\left\|E_{\mathrm{s}} h\right\| \leqq \\
& \leqq 5^{n} \cdot 16 \cdot 3^{4 n} C_{1}^{2} C_{2}^{2} m^{2}|t|^{2 n} \sum_{\mathrm{r}}\left\|E_{\mathbf{r}} h\right\|^{2} .
\end{aligned}
$$

Since $Q$ is hermitian we may conclude that

$$
\|Q\| \leqq 4 \cdot 9^{n} \cdot 5^{n / 2} C_{1} C_{2} m|t|^{n}\|h\| .
$$

Recalling that $Q=Q(\alpha)$ depends on $\alpha$ (equation $(1 \mathrm{~b})$ ) we note that $Z=Q(1)-i Q(i)$. Therefore the norm of $Z$ is bounded by the expression,

$$
\|Z(h, g, f ; t)\| \leqq 8 \cdot 9^{n} \cdot 5^{n / 2} m C_{1} C_{2}|t|^{n}\|h\|
$$

Hence, the lemma is proved.

Lemma 2. Suppose $\psi$ is a relativistic field satisfying assumptions $I-I V$. Let $\mathfrak{S}_{0}$ be the linear manifold obtained by applying all polynomials in the smeared fields at time, $t=0$, to the vacuum state, $\Omega_{0}$, i.e.,

$$
\mathfrak{D}_{0}=\left\{\Omega ; \Omega=p\left(\psi\left(f_{1}, 0\right)^{\#}, \ldots, \psi\left(f_{k}, 0\right)^{\#}\right) \Omega_{0}\right\} .
$$

Then, if $f, g \in \mathfrak{T}$, the operator,

$$
B(g, f ; t)=\left\{\psi(g, 0)^{\#}, \frac{\psi(f, t) \#-\psi(f, 0)^{\#}}{t}\right\}_{+},
$$

converges strongly to a c-number on $\mathfrak{S}_{0}$ as $t \rightarrow 0$.

Proof. Clearly, we have $\psi(f, 0)^{\#} \mathfrak{S}_{0} \subset \mathfrak{S}_{0}$ for all $f \in \mathfrak{G}_{T}$. Since the field operators, $\psi(f, 0)^{\#}$, act irreducibly on $\mathfrak{H}, \mathfrak{D}_{0}$ is dense in $\mathfrak{G}$.

From lemma 1 it follows that

$$
\left\|\left[\psi(h, 0)^{\#}, B(g, f ; t)\right] \_\right\| \leqq C|t|^{n-1}\|h\|,
$$

where the constant, $C$, depends only on the functions, $f, g \in \mathfrak{T}$.

It follows that if $P=p\left(\psi\left(f_{1}, 0\right)^{\#}, \ldots, \psi\left(f_{k}, 0\right)^{\#}\right)$ is a polynomial in the smeared fields at time, $t=0$, then

$$
\left\|[P, B(g, f ; t)] \_\right\| \leqq C K(p)|t|^{n-1},
$$

where $K(p)$ is a constant depending only on the polynomial, $P$. Since by assumption, $n>1$, the right hand side of the above inequality converges to zero as $t \rightarrow 0$. Therefore, $B(g, f ; t)$ converges on every vector, $\Omega \in \mathfrak{D}_{0}$, if it converges on the vacuum, since

$$
\left\|P B(g, f ; t) \Omega_{0}-B(g, f ; t) P \Omega_{0}\right\| \rightarrow 0 \quad \text { as } t \rightarrow 0 .
$$

However, assumption IV assures us that $B(g, f ; t) \Omega_{0}$ converges strongly as $t \rightarrow 0$ and, hence, $B(g, f ; t)$ converges strongly on $\mathfrak{S}_{0}$. We define the limiting operator, $B_{0}(g, f)$ on $\mathfrak{S}_{0}$ with the relation,

$$
B_{0}(g, f) \Omega=\lim _{t \rightarrow 0} B(g, f ; t) \Omega \text { all } \Omega \in \mathfrak{S}_{0} .
$$


Since $B(g, f ; t)^{*}$ has the same form as $B(g, f ; t)$ (when expressed in terms of the fields, $\left.\psi^{\#}\right), B(g, f ; t)^{*}$ also converges strongly on $\mathfrak{S}_{0}$ as $t \rightarrow 0$. Let $B_{0}^{\prime}(g, f)$ be the linear operator defined on $\mathfrak{S}_{0}$ by the relation,

$$
B_{0}^{\prime}(g, f) \Omega=\lim _{t \rightarrow 0} B(g, f ; t) * \Omega \text {, all } \Omega \in \mathfrak{D}_{0} .
$$

It follows immediately from the definition of $B_{0}$ and $B_{0}^{\prime}$ that

$$
\left(\Omega_{1}, B_{0}(g, f) \Omega_{2}\right)=\left(B_{0}^{\prime}(g, f) \Omega_{1}, \Omega_{2}\right) \text {, all } \Omega_{1}, \Omega_{2} \in \mathfrak{S}_{0} .
$$

Hence, the adjoint of $B_{0}(g, f)$ is densely defined, (in fact, $\left.B_{0}(g, f)^{*}\right)$ $\left.\supset B_{0}^{\prime}(g, f)\right)$ so $B_{0}(g, f)$ has a unique extension to a closed operator, $\overline{B_{0}(g, f)}$. From inequality $(2 a)$ it follows that $\overline{B_{0}(g, f)}$ commutes with the fields at time, $t=0$, on $\mathfrak{S}_{0}$, i.e.,

$\overline{B_{0}(g, f)} \psi(h, 0)^{\#} \Omega=\psi(h, 0)^{\#} \overline{B_{0}(g, f)} \Omega \quad$ for $\quad h \in \mathfrak{H}_{T} \quad$ and $\quad \Omega \in \mathfrak{S}_{0}$.

This equation can be extended to all vectors, $\Omega \in \mathfrak{F}$, which are in the domain of $\overline{B_{0}(g, f)}$, since $\overline{B_{0}(g, f)}$ is closed and $\psi(h, 0)^{\#}$ is a bounded operator. Since the fields, $\psi(f, 0)^{\#}$, act irreducibly on $\mathfrak{G}$, it follows from the generalized Schur lemma $[6]$ that $\overline{B_{0}(g, f)}$ is a $c$-number. On $\mathfrak{D}_{0}$, $B(g, f ; t)$ converges strongly to $\overline{B_{0}(g, f)}$, so the lemma is proved.

Lemma 3. There exist a linear operator, $T_{1}$, and an antilinear operator, $T_{2}$, defined on $\mathfrak{T}$ such that, for all $f, g \in \mathfrak{T}$, the operators,

$$
\begin{aligned}
& A(t)=\left\{\psi(g, 0)^{\#},\left(\frac{\psi(f, t)-\psi(f, 0)}{t}\right)-\psi\left(T_{1} f, 0\right)-\psi\left(T_{2} f, 0\right)^{*}\right\}_{+} \\
& B(t)=\left\{\psi(g, 0)^{\#},\left(\frac{\psi(f, t)^{*}-\psi(f, 0)^{*}}{t}\right)-\psi\left(T_{1} f, 0\right)^{*}-\psi\left(T_{2} f, 0\right)\right\}_{+}
\end{aligned}
$$

converge strongly to zero on $\mathfrak{S}_{0}$ as $t \rightarrow 0$.

Proof. We define the operator, $T_{1}$, by the relation,

$$
\left(g, T_{1} f\right)=\lim _{t \rightarrow 0}\left(\Omega_{0},\left\{\psi(g, 0)^{*}, \frac{(\psi(f, t)-\psi(f, 0)}{t}\right\}_{+} \Omega_{0}\right) .
$$

From assumption IV it follows that this limit exists, provided $f \in \mathfrak{T}$, and furthermore,

$$
\begin{aligned}
\left|\left(g, T_{1} f\right)\right| & \leqq\left\|\psi(g, 0)^{*}\right\|\left(\left\|\partial_{t} \psi(f, 0) \Omega_{0}\right\|+\left\|\partial_{t} \psi(f, 0)^{*} \Omega_{0}\right\|\right) \\
& \leqq\|g\|\left(\left\|\partial_{t} \psi(f, 0) \Omega_{0}\right\|+\left\|\partial_{t} \psi(f, 0)^{*} \Omega_{0}\right\|\right) .
\end{aligned}
$$

Then, $\left(g, T_{1} f\right)$ is, for each fixed $f \in \mathfrak{T}$, a norm continuous antilinear functional in $g$. Hence, by a theorem due to F. Riesz [7], there is a unique vector, $h \in \mathfrak{S}_{T}$, such that $\left(g, T_{1} f\right)=(g, h)$, so $T_{1} f=h$. Clearly, $T_{1}$, defined in this way, is a linear operator from $\mathfrak{T}$ into $\mathfrak{G}_{T}$. The antilinear operator, $T_{2}$, is similarly defined by the relation,

$$
\left(T_{2} f, g\right)=\lim _{t \rightarrow 0}\left(\Omega_{0},\left\{\psi(g, 0), \frac{(\psi(f, t)-\psi(f, 0)}{t}\right\}_{+} \Omega_{0}\right),
$$


$T_{2}$ is an antilinear operator from $\mathfrak{T}$ into $\mathfrak{S}_{T}$. It follows from lemma 2 that $A(t)$ and $B(t)$ converge strongly to a $c$-number on $\mathfrak{S}_{0}$ as $t \rightarrow 0$ (since $A(t)$ and $B(t)$ differ from $B(g, f ; t)$ by a $c$-number). From the definitions of $T_{1}$ and $T_{2}$ it follows that the expectation values, $\left(\Omega_{0}, A(t) \Omega_{0}\right)$ and $\left(\Omega_{0}, B(t) \Omega_{0}\right)$ converge to zero as $t \rightarrow 0$. Hence $A(t)$ and $B(t)$ converge strongly to zero on $\mathfrak{S}_{0}$ as $t \rightarrow 0$. And the lemma is proved.

Remark. It follows from the CAR and the translation invariance of the vacuum, $\Omega_{0}$, that the vacuum expectation of odd monomials in the smeared fields vanishes [8], i.e.,

$$
\left(\Omega_{0}, \psi\left(f_{1}, t_{1}\right)^{\#} \ldots \psi\left(f_{n}, t_{n}\right)^{\#} \Omega_{0}\right)=0, \text { for } n \text { odd . }
$$

Therefore, the mapping, $\psi(f, t)^{\#} \rightarrow-\psi(f, t)^{\#}$, is a symmetry transformation of the field. Since the CAR and vacuum expectation values are preserved under this mapping, there exists a unitary operator, $U_{I}$, which implements this transformation. The unitary operator, $U_{I}$, has the properties,

$$
\begin{gathered}
U_{I} \Omega_{0}=\Omega_{0} \\
U_{I} \psi(f, t)^{\#} U_{I}^{-1}=-\psi(f, t)^{\#} .
\end{gathered}
$$

Since $U_{I}^{2}$ commutes with the fields, $\psi(f, t)^{\#}$, it follows that $U_{I}^{2}=\alpha I$. And the fact that $U_{I}$ leaves the vacuum invariant implies that $\alpha=1$. Therefore, $U_{I}$ is hermitian. We will make use of the operator, $U_{I}$, in the following lemma.

Lemma 4. Let $\mathfrak{S}_{1}$ be the linear manifold obtained by applying all polynomials in the smeared fields, $\psi(f, 0)^{\#}$, with $f \in \mathfrak{T}$, to the vacuum, i.e.,

$$
\mathfrak{S}_{1}=\left\{\Omega ; \Omega=p\left(\psi\left(f_{1}, 0\right)^{\#}, \ldots, \psi\left(f_{r}, 0\right)^{\#}\right) \Omega_{0}, f_{1}, \ldots, f_{r} \in \mathfrak{T}\right\} .
$$

Let $T_{1}$ and $T_{2}$ be the operators defined in lemma 3 . Then, if $\psi$ is a field satisfying assumptions $I-I V$, the operator,

$$
Q(f, t)=\frac{\psi(f, t)-\psi(f, 0)}{t}-\psi\left(T_{1} f, 0\right)-\psi\left(T_{2} f, 0\right)^{*},
$$

converges strongly to zero on $\mathfrak{D}_{1}$ as $t \rightarrow 0$.

Proof. Let $U_{I}$ be the unitary operator discussed in the preceeding remarks. We will show that the operator, $U_{I} Q(f, t)$, converges strongly to a $c$-number on $\mathfrak{D}_{1}$. The commutator of $U_{I} Q(f, t)$ with the smeared fields can be written in the form,

$$
\left[\psi(g, 0)^{\#}, U_{I} Q(f, t)\right]_{-}=-U_{I}\left\{\psi(g, 0)^{\#}, Q(f, t)\right\}_{+} .
$$

It follows from lemma 3 that this commutator converges strongly to zero on $\mathfrak{S}_{0}$ as $t \rightarrow 0$, provided $t, g \in \mathfrak{T}$, i.e.,

$$
\left\|\psi(g, 0)^{\#} U_{I} Q(f, t) \Omega-U_{I} Q(f, t) \psi(g, 0)^{\#} \Omega\right\| \rightarrow 0 \quad \text { as } t \rightarrow 0,
$$
if $\Omega \in \mathfrak{S}_{0} \supset \mathfrak{S}_{1}$ and $f, g \in \mathfrak{T}$. 
By assumption IV $U_{I} Q(f, t) \Omega_{0}$ converges strongly to a limit as $t \rightarrow 0$. It follows from equation (4a) that $U_{I} Q(f, t) \psi(g, 0)^{\#} \Omega_{0}$ converges strongly as $t \rightarrow 0$. Using equation (4a) by induction, one sees that the expression,

$$
U_{I} Q(f, t) \psi\left(g_{1}, 0\right)^{\#} \ldots \psi\left(g_{r}, 0\right)^{\#} \Omega_{0}, \quad g_{1}, \ldots, g_{r} \in \mathfrak{T},
$$

converges strongly to a limit as $t \rightarrow 0$. Hence, $U_{I} Q(f, t)$ converges strongly on $\mathfrak{D}_{1}$. Proceeding as in lemma 2 , we define the linear operator, $A(f)$, on $\mathfrak{D}_{1}$ by the relation,

$$
A(f) \Omega=\lim _{t \rightarrow 0} U_{I} Q(f, t) \Omega, \quad \Omega \in \mathcal{O}_{1} .
$$

Next we argue that the operator, $\left(U_{I} Q(f, t)\right)^{*}=Q(f, t)^{*} U_{I}$, converges strongly on $\mathfrak{S}_{1}$ as $t \rightarrow 0$. Clearly, we have $U_{I} \mathfrak{D}_{1} \subset \mathfrak{S}_{1}$. The commutator of $\left(U_{I} Q(f, t)\right)^{*}$ with the fields at time, $t=0$, can be written

$$
\left[\psi(g, 0)^{\#},\left(U_{I} Q(f, t)\right)^{*}\right]_{-}=\left\{\psi(g, 0)^{\#}, Q(f, t)^{*}\right\}_{+} U_{I} .
$$

By assumption IV we know that $\left(U_{I} Q(f, t)\right) * \Omega_{0}=Q(f, t) * \Omega_{0}$ converges strongly to a limit as $t \rightarrow 0$. And from lemma 3 we know that the above commutator converges strongly to zero on $\mathfrak{D}_{0}$ as $t \rightarrow 0$, provided $g \in \mathfrak{T}$. Therefore, by the argument used previously, the operator $\left(U_{I}(Q f, t)\right)^{*}$, converges strongly on $\mathfrak{D}_{1}$. We define $A^{\prime}(f)$ on $\mathfrak{S}_{1}$ by the relation,

$$
A^{\prime}(f) \Omega=\lim _{t \rightarrow 0}\left(U_{I} Q(f, t)\right)^{*} \Omega, \quad \Omega \in \mathfrak{S}_{1} .
$$

From the definition of $A(f)$ and $A^{\prime}(f)$ it follows that

$$
\left(\Omega_{1}, A(f) \Omega_{2}\right)=\left(A^{\prime}(f) \Omega_{1}, \Omega_{2}\right), \quad \Omega_{1}, \Omega_{2} \in \mathfrak{D}_{1} .
$$

Since the adjoint of $A(f)$ is densely defined, $A(f)$ can be uniquely extended to a closed operator, $\overline{A(f)}$. From relation $(4 \mathrm{a})$ it follows that $\overline{A(f)}$ commutes with the fields, $\psi(f, 0)^{\#}$, with $f \in \mathcal{T}$. Due to the facts that $\mathfrak{T}$ is dense in $\mathfrak{G}_{T}$, the fields, $\psi(f, 0)^{\#}$, are bounded by the $L^{2}$-norm of the test function, and $\overline{A(f)}$ is a closed operator, it follows that $\overline{A(f)}$ commutes with the fields, $\psi(f, 0)^{\#}$, for all $f \in \mathfrak{G}_{T}$. Since the fields, $\psi(f, 0)^{\#}$, act irreducibly on $\mathfrak{G}$, it follows from the generalized Schur lemma [6] that $\overline{A(f)}$ is a $c$-number. On $\mathfrak{D}_{1}, U_{I} Q(f, t)$ converges strongly to $\overline{A(f)}$, so $U_{I} Q(f, t)$ converges strongly to a $c$-number on $\mathfrak{D}_{1}$. To evaluate this $c$-number we consider the vacuum expectation,

$$
\lim _{t \rightarrow 0}\left(\Omega_{0}, U_{I} Q(t, t) \Omega_{0}\right)=\lim _{t \rightarrow 0}\left(\Omega_{0}, Q(f, t) \Omega_{0}\right)=0 .
$$

The limit is zero since the vacuum expectation of odd monomials in the fields vanishes. Hence, $U_{I} Q(f, t)$ converges strongly to zero on $\mathfrak{D}_{1}$ as $t \rightarrow 0$. Since $U_{I}$ is unitary, $Q(f, t)$ converges strongly to zero on $\mathfrak{D}_{1}$. And the lemma is proved. 
Lemma 5. If $\psi$ is a Fermi field satisfying assumptions $I-I V$, then $\psi$ satisfies the differential equation,

$$
\partial_{t} \psi(f, t)=\psi\left(T_{1} f, t\right)+\psi\left(T_{2} f, t\right)^{*},
$$

where $T_{1}$ and $T_{2}$ are, respectively, linear and antilinear operators defined on $\mathfrak{T}$. The time derivative of the field, $\partial_{t} \psi(f, t)$, exists for all $f \in \mathfrak{T}$, in the sense of strong convergence, i.e.,

$$
\lim _{h \rightarrow 0} \frac{\psi(f, t+h)-\psi(f, t)}{h} \Omega=\partial_{t} \psi(f, t) \Omega, \text { for all } \Omega \in \mathfrak{G} .
$$

Proof. Suppose $f \in \mathfrak{T}$ is an $m$-component function with support in the half space, $\{\mathbf{x} ; \mathbf{x} \cdot \mathbf{n}<C, \mathbf{n} \neq 0\}$. Let $Q(f, t)$ be defined as in lemma 4 . We know from lemma 4 that

$$
\left\|Q(f, t) \Omega_{0}\right\| \rightarrow 0, \text { as } t \rightarrow 0 .
$$

We will make use of the transformation law of the fields and the invariance of the vacuum under the action of the time translation operators, $U(t)$;

$$
\begin{gathered}
U\left(t_{1}\right) \psi\left(f, t_{2}\right)^{\#} U\left(t_{1}\right)^{-1}=\psi\left(f, t_{1}+t_{2}\right)^{\#}, \\
U(t) \Omega_{0}=\Omega_{0}, \quad \text { for all } t .
\end{gathered}
$$

It follows from relation (5a) that

$$
\left\|\int_{t_{1}}^{t_{2}} d t U(t) Q(f, h) \Omega_{0}\right\| \rightarrow 0, \text { as } h \rightarrow 0 .
$$

Using equations (5b) and (5c) and the definition of $Q(f, h)$, we can rewrite this relation in the form,

$$
\begin{aligned}
\|\left(h^{-1} \int_{0}^{h} d t\left(\psi\left(f, t_{2}+t\right)-\psi\left(f, t_{1}+t\right)\right)-\right. & \\
& -\int_{t_{1}}^{t_{2}} d t\left(\psi\left(T_{1}(f, t)+\psi\left(T_{2} f, t\right)^{*}\right)\right) \Omega_{0} \| \rightarrow 0 \text { as } h \rightarrow 0 .
\end{aligned}
$$

Since $U(t)$ is strongly continuous in $t$, we obtain in the limit of $h \rightarrow 0$ the equation,

$$
\left(\psi\left(f, t_{2}\right)-\psi\left(f, t_{1}\right)-\int_{t_{1}}^{t_{2}} d t\left(\psi\left(T_{1} f, t\right)+\psi\left(T_{2} f, t\right)^{*}\right)\right) \Omega_{0}=A \Omega_{0}=0 .
$$

Clearly, $A$ is a bounded operator associated with a space-time region, $\mathcal{O}$. By assumption, the function, $f$, has support in the half-space, $\{\mathbf{x} ; \mathbf{x} \cdot \mathbf{n}<C\}$. The range of integration is finite. Therefore, the set $\mathfrak{O}^{\prime}$ of space-time points which are space-like separated from every point of $\mathfrak{O}$, is non-empty. Since $A$ annihilates the vacuum, it follows from a theorem due to ReEH and Schlieder [9], that $A=0$. Hence, we have

$$
\frac{\psi(f, t+h)-\psi(f, t)}{h}=\frac{1}{h} \int_{t}^{t+h} d t^{\prime}\left(\psi\left(T_{1} f, t^{\prime}\right)+\psi\left(T_{2} f, t^{\prime}\right)^{*}\right) .
$$


for all $f \in \mathfrak{T}$ with support in a half-space. This equation can be extended to all $f \in \mathbb{T}$, due to the linearity of this equation in the test function, $f$. The right-hand side of the above equation converges strongly to $\psi\left(T_{1} f, t\right)+\psi\left(T_{2} f, t\right)^{*}$, as $h \rightarrow 0$. Hence, the lemma is proved.

Acknowledgements. The author is grateful for discussions with Professor H. Borchers, A. Jaffe, O. Landford, and Professor A. Wightman. The author is grateful for a National Science Foundation Graduate Fellowship.

\section{References}

[1] Källén, G.: Kgl. Danske Videnskab. Selskab, Mat.-Fys. Medd. 27, 12 (1953).

[2] Kladder, J. R.: J. Math. Phys. 6, 1666 (1965).

[3] Streater, R. F., and A. S. Wightman: PCT, spin and statistics and all that, Chap. III. New York: W. A. Benjamin, Inc. 1964.

[4] Araki, H., and W. Wyss: Helv. Phys. Acta 37, 136 (1964).

[5] Schweber, S. S.: An introduction to relativistic quantum field theory, Chap. 17, p. 674. New York: Harper and Row 1962.

[6] Naimark, M. A.: Normed rings, Chap. IV, section 21. Groningen, the Netherlands: P. Noordhoff N. V. 1964.

[7] Riesz, F., and B. Sz.-Nagy: Functional analysis, Chap. II, section 30. New York: Ungar 1955.

[8] Streater, R. F., and A. S. Wightman: Ref. 3, p. 152-153.

[9] - Ref. 3, p. 139. 